\title{
De la femme aux individus : l'histoire du genre en Grande-Bretagne,des années 1960 à nos jours
}

From women's history to the history of subjectivity: gender history in Great

Britain, 1960s to the present

\section{Christina de Bellaigue}

\section{(2) OpenEdition}

Journals

Édition électronique

URL : http://journals.openedition.org/rh19/3510

DOI : $10.4000 /$ rh 19.3510

ISSN : $1777-5329$

Éditeur

La Société de 1848

Édition imprimée

Date de publication : 15 novembre 2008

Pagination : 55-69

ISSN : 1265-1354

Référence électronique

Christina de Bellaigue, « De la femme aux individus : I'histoire du genre en Grande-Bretagne,des années 1960 à nos jours », Revue d'histoire du XIXe siècle [En ligne], 37 | 2008, mis en ligne le 01 décembre 2010, consulté le 30 avril 2019. URL : http://journals.openedition.org/rh19/3510 ; DOI $10.4000 /$ rh19.3510 


\section{CHRISTINA DE BELLAIGUE}

\section{De la ferme aux individus : l'histoire du genre en Grande-Bretagne, des années 1960 à nos jours}

2007 a marqué le vingtième anniversaire de la parution de l'ouvrage de Leonore Davidoff et Catherine Hall, Family Fortunes : men and women of the English middle class, 1780-1830 ${ }^{1}$. Ce livre a été considéré comme «l'ouvrage ayant eu le plus d'influence sur l'histoire du genre en Grande-Bretagne» ${ }^{2}$. On pourrait même affirmer que Family Fortunes est parvenu à atteindre l'un des objectifs des historiennes féministes des années 1960 : non seulement exhumer l'expérience féminine et un passé longtemps ignoré, mais aussi remettre en cause les certitudes d'une histoire politique, sociale et économique qui ignorait alors le genre, les rapports sociaux de sexe et les identités masculine et féminine.

En effet, Family Fortunes parut à un moment de transition important dans l'histoire du genre en Grande-Bretagne. Sa publication en 1987 marqua en quelque sorte l'apogée (mais non la fin) de l'entreprise collective, inspirée par la tradition féministe socialiste, qui tentait de retrouver les voix et les expériences de celles qui étaient, comme le disait Sheila Rowbotham, «ignorées de l'histoire " ${ }^{3}$. En même temps, Family fortunes précédait, et d'une certaine manière anticipait, un nouveau champ de recherche s'intéressant particulièrement à la représentation, aux discours de la masculinité et de la féminité, ainsi qu'à l'importance et à l'empreinte que ceux-ci pouvaient laisser. Sous l'influence de cette réorientation, on passait d'une période où l'histoire des femmes n'attirait guère que l'attention de spécialistes et de celles qui y voyaient un élément important de la lutte féministe, à une époque où l'histoire du genre devint couramment un sujet d'articles publiés dans les périodiques canoniques de l'historiographie britannique, tels que le Histo-

1. Leonore Davidoff et Catherine Hall, Family fortunes: men and women of the English middle class, 17801830, Londres, Routledge, 2002 (1987).

2. Kathryn Gleadle, "Revisiting Family Fortunes: reflections on the twentieth anniversary of the publication of L. Davidoff \& C. Hall (1987) Family Fortunes: Men and Women of the English Middle Class, 1780-1850 (Londres: Hutchinson)", Women's History Review, tome 16, n 5, 2007, p. 773-782.

3. Sheila Rowbotham, Hidden from History: 300 Years of Women's Oppression and the Fight against it, Londres, Pluto Press, 1977 (1973). L'auteure est responsable de toutes les traductions de l'anglais; plutôt que l'élégance de l'expression, on a cherché à restituer le plus fidèlement possible le sens de l'original. 
rical Journal et Past and Present. Ainsi, par exemple, entre 1960 et 1989, le Historical Journal ne publia que 28 articles traitant explicitement d'une façon ou une autre du "gender»; entre 1990 et 2002, il en publia 181. En 2008, le genre est devenu une catégorie d'analyse courante dans tous les domaines historiques. De leur côté, les chercheurs spécialisés dans l'histoire du genre, tout en reconnaissant toujours son caractère construit, et la multiplicité des conceptions de la masculinité et de la féminité, tentent de mieux comprendre comment ces catégories agissent sur le vécu de l'individu, et sont modifiées par lui.

\section{IGNORÉES DE L'HISTOIRE}

Sous la double impulsion du féminisme et de la «nouvelle histoire sociale» ("New Social History») inspirée par les idées d'E.P. Thompson entre autres, les années 1960 et 1970 virent se multiplier les ouvrages qui tentaient de retrouver les expériences des femmes du passé. En particulier, l'histoire des femmes de la classe ouvrière et du féminisme suscitait l'intérêt d'une génération de chercheuses travaillant souvent aux marges de la profession historique. Enseignant dans les collèges d'enseignement pour adultes, dans les départements de sociologie et d'éducation, plutôt que dans les facultés d'histoire universitaires, ces historiennes se rattachaient souvent à un mouvement féministe marxisant. Elles soutenaient que la conscience du passé soulignait le caractère construit des relations entre les sexes et de l'oppression féminine. L'influence du marxisme mena à une réflexion qui tentait de rattacher l'histoire des femmes et du genre à l'histoire des classes sociales et du travail. Pour Sheila Rowbotham, l'objectif principal était de se demander «dans quelles conditions les femmes [ont] produit et reproduit leur vie, et par leur travail et par la procréation " 4 . On prendra deux exemples pour montrer comment ces influences ont pu inspirer une histoire des femmes politiquement engagée, qui commençait à remettre en question les certitudes de l'historiographie britannique.

D'abord, l'ouvrage de Jill Liddington et Jill Norris, One Hand Tied Behind Us: the Rise of the Women's Suffrage Movement ${ }^{5}$. Travaillant toutes deux dans l'enseignement pour adultes à Manchester, Jill Liddington et Jill Norris puisèrent dans les archives locales et profitèrent de leurs liens avec la communauté ouvrière qui les entourait pour exhumer l'histoire des associations suffragistes du Lancashire à la fin du XIX ${ }^{\mathrm{e}}$ siècle et au début du $\mathrm{XX}^{\mathrm{e}}$ siècle. Elles découvrirent ainsi l'expérience d'un groupe de femmes très différent des féministes londoniennes qui tournaient autour de Milicent

4. Idem, p. ix.

5. Jill Liddington et Jill Norris, One Hand Tied Behind Us: the Rise of the Women's Suffrage Movement, Londres, Rivers Oram Press, 2000 (1978). 
Garrett Fawcett, d'Emmeline Pankhurst et de la Women's Social and Political Union (WSPU), les «suffragettes» qui avaient jusqu'alors retenu toute l'attention des historiens. Non seulement les «suffragistes» du Lancashire étaient issues d'un milieu très différent de celui des dames de la WSPU - qui étaient recrutées pour la plupart dans les classes moyennes et les professions libérales - mais leurs méthodes et leurs objectifs étaient différents. En 1906, Eva Gore-Booth, un personnage important de ce mouvement à Manchester, écrivait à Milicent Fawcett pour protester au nom du profil type des membres de son association contre les méthodes militantes de la WSPU : «ce ne sont pas les manifestations, ni même la violence qui lui répugnent, c'est le fait d'être confondu et tenu responsable en tant que classe pour les actions de dames instruites et fortunées qui donnent des coups de pied, qui crient, qui mordent et qui crachent ${ }^{6}$. Elle exprimait ainsi une conscience de la situation particulière des féministes ouvrières qui se révélait également dans leurs objectifs. Celles-ci s'intéressaient au vote non seulement comme un droit qui leur était dû, mais aussi comme un moyen d'assurer les améliorations sociales et économiques qui leur semblaient essentielles. C'est un thème repris dans le titre de l'ouvrage, extrait d'un discours de Hannah Mitchell : obligées de travailler et de maintenir leurs foyers, ces féministes ouvrières menaient leur campagne, selon elle, avec "une main attachée dans le dos" 7 . Soulignant l'interaction de la conscience de classe avec la conscience du genre, One Hand Tied Behind Us fit découvrir et revivre les voix et les expériences de femmes jusqu'alors oubliées.

Pour Sally Alexander, il était également important de combler les lacunes d'une histoire souvent ignorante de l'expérience des femmes. À un moment $\mathrm{du} \mathrm{XX}^{\mathrm{e}}$ siècle où l'émancipation féminine semblait se rattacher étroitement au droit au travail, à l'égalité professionnelle et à l'indépendance économique, Sally Alexander s'intéressa à l'histoire du travail des femmes au XIX ${ }^{\mathrm{e}}$ siècle. Elle exhuma l'histoire négligée des couturières, des lingères, des relieuses, des coiffeuses et des petites manufactures au personnel féminin ${ }^{8}$. Ce faisant, elle montrait avec éclat l'impact que l'histoire des femmes peut avoir sur notre compréhension de l'histoire économique. Son étude faisait apparaître que ce n'était pas seulement l'évolution économique, technique et industrielle, ni l'invention de nouveaux modes de production, qui menait à la hausse de productivité que l'on nomme la révolution industrielle, mais surtout l'essor du travail des femmes et des enfants. Mal payée, sans protection syndicale, cette main-d'œuvre était idéale pour une période d'expansion commerciale. En même temps, Sally Alexander remettait en cause toute notion d'une division «naturelle» du travail entre les sexes. Son étude sur le travail des femmes

6. Idem, p. 210.

7. Idem, p. 6.

8. Sally Alexander, "Women's work in nineteenth-century London", dans Juliet Mitchell et Ann Oakley (eds.), The Rights and Wrongs of Women, Londres, Penguin, 1976, p. 17-58. 
à Londres révélait comment avec chaque nouvelle technologie, la division sexuelle des tâches se remaniait, se renégociait, toujours selon une hiérarchie qui dévalorisait le travail féminin.

Selon Sally Alexander, la reconnaissance de cette continuité d'un système d'oppression patriarcale était essentielle. En revanche, pour les historiens des années 1980, il était plus important d'expliquer comment les rapports sociaux de sexe, les identités masculines et féminines, se forment et se construisent à chaque moment ${ }^{9}$. Pour ceux-ci, le genre devint la catégorie d'analyse principale, et celle dont ils espéraient qu'elle contribuerait à une réévaluation fondamentale des processus de l'histoire sociale, politique, économique et culturelle.

\section{LA FAMILLE ET LA FORTUNE}

Ces larges ambitions trouvèrent une expression importante dans l'œuvre de Leonore Davidoff et Catherine Hall. "Nous voulions, expliquait Catherine Hall en 1992, non seulement replacer les femmes dans l'histoire dont elles avaient été exclues, mais réécrire cette histoire pour qu'on reconnaisse la façon dont le genre, un des principaux axes du pouvoir dans la société, fournit un élément essentiel de la structure et de l'organisation sociale» ${ }^{10}$. Au cœur de leur ouvrage, on trouve donc l'hypothèse que la formation des classes se rattache à la construction d'identités sexuées, qu'elle en dépend même. Leur thèse reposait sur une étude approfondie de la formation de la middle class provinciale dans le Norfolk, le Suffolk et à Birmingham. S'appuyant sur les recensements de population, sur des archives privées de familles bourgeoises, sur les sermons et les manuels de savoir-vivre qui se multipliaient à la fin du XVIII ${ }^{e}$ siècle, elles avançaient une thèse novatrice : c'est l'idéologie de la domesticité qui était au cœur de l'identité de la middle class en Angleterre, et qui la rendit si puissante dans l'économie, dans la politique, et dans le domaine culturel, au XIX ${ }^{\mathrm{e}}$ siècle.

Selon Leonore Davidoff et Catherine Hall, cette idéologie de la domesticité avait ses racines dans la séparation de plus en plus rigide entre une sphère publique (masculine) et une sphère privée (féminine) qui se faisait sentir depuis la fin du XVIII siècle. Cette idéologie s'inspirait de la pensée du renouveau évangélique. En réponse à l'émergence d'un mode de croissance industrielle dominé par les principes de concurrence commerciale, les idéologues de ce renouveau - William Cowper, Hannah More, John Angell James - donnaient au foyer familial un caractère pieux et sacré. La

9. Sally Alexander et Barbara Taylor, «In defence of 'patriarchy'”, dans Raphael Samuel (ed.), People's History and Socialist Theory, Londres, Routledge and Kegan, 1981, p. 370-373.

10. Catherine Hall, White, Male and Middle-class: Explorations in Feminism and History, Londres, Polity, 1992, p. 12-13. 
femme, être domestique par nature et par vocation, était au cœur de ce foyer ("home») idéal. Dépendant complètement de son mari, elle élevait ses enfants dans la piété et la moralité et se dévouait entièrement à conserver le foyer où les hommes de la famille pouvaient trouver un sanctuaire et échapper aux influences négatives de la vie publique et du commerce. C'est à l'homme qu'il incombait de rendre possible la réalisation de cet idéal par son travail et par la protection du patrimoine de la famille. Comme le montraient Leonore Davidoff et Catherine Hall, cette idéologie de la domesticité était renforcée par le processus d'industrialisation et d'urbanisation qui créait de plus en plus de distance entre le foyer familial et le lieu de travail. Sous l'impulsion de cet idéal de la domesticité, les villes anglaises se dotaient d'une banlieue de petits pavillons où chacun pouvait recréer son idylle domestique pseudorurale, loin des réalités brutales de la vie commerciale.

L'idée que le modèle des sphères séparées déterminait les rapports de sexe au XIX ${ }^{e}$ siècle n'était pas nouvelle en 1987. C'était même un concept de plus en plus contesté par les historiens des femmes et du genre : dans un article publié aux États-Unis en 1988, Linda Kerber estimait que les chercheurs se montraient si fidèles à cette notion qu'ils contribuaient à reconstruire constamment les mêmes distinctions que leurs prédécesseurs victoriens ${ }^{11}$. En Grande-Bretagne, en 1993, Amanda Vickery critiqua avec force les conclusions de Leonore Davidoff et Catherine Hall, fondées, selon elle, sur une lecture erronée de la littérature prescriptive : les ouvrages de Hannah More, de Cowper et des autres écrivains auxquels Family fortunes faisait référence ne pouvaient être lus comme décrivant la vie réelle des femmes. Au contraire, ces publications se multipliaient précisément parce qu'en réalité, la vie des femmes se conformait de moins en moins à l'idéal des sphères séparées ${ }^{12}$.

Cependant, une relecture attentive de l'ouvrage de Leonore Davidoff et Catherine Hall montre que celles-ci étaient moins convaincues de la réalité des sphères séparées que ne le pensaient leurs critiques. En fait, elles insistaient précisément sur le fait que «la sphère publique n'était pas véritablement publique et le domaine privé n'était pas entièrement privé, malgré la métaphore des sphères séparées» ${ }^{13}$. Par ailleurs, à la différence des chercheurs qui les précédaient, qui voyaient surtout dans le concept des «sphères séparées" un mécanisme de la subordination des femmes, Leonore Davidoff et Catherine Hall soulignaient la façon dont cette idéologie pouvait exercer une influence positive. Ainsi, elles notaient comment l'insistance de Hannah More pour dire que «la vocation professionnelle des femmes» était de se marier et de devenir mère de famille valorisait l'expérience féminine et

11. Linda Kerber, "Separate spheres, female worlds, a woman's place: the rhetoric of women's history", Journal of American History, tome 75, $\mathrm{n}^{\circ}$ 1, 1988, p. 9-39.

12. Amanda Vickery, "Golden age to separate spheres? A review of the categories and chronologies of women's history", Historical Journal, vol. 36, n 2, 1993, p. 383-414.

13. Leonore Davidoff et Catherine Hall, Family Fortunes...,ouv. cité, p. 33. 
contribuait à renforcer les arguments de ceux qui souhaitaient étendre l'éducation proposée aux filles. Ce sont en partie cette reconnaissance de la fluidité entre public et privé et la prise de conscience de la séduction de l'idéologie domestique, qui firent la force de Family Fortunes.

On retrouve l'influence de leur travail dans plusieurs domaines de l'histoire sociale et culturelle. Des recherches pointues dans les archives privées comme celles de la famille Courtauld - dont les manufactures de soie firent la fortune de sa deuxième génération - poussèrent les auteurs de Family Fortunes à souligner la contribution importante des femmes au financement et aux effectifs de l'entreprise familiale. Leonore Davidoff et Catherine Hall montrèrent comment les mères, les sœurs, les épouses et les veuves, de plus en plus éloignées de la gestion quotidienne de l'entreprise familiale et privées des moyens d'agir indépendamment par les lois limitant l'accès des femmes à la propriété, contribuaient néanmoins, par leur «investissement caché», au succès de l'entreprise. C'était une découverte importante pour les historiens de l'entreprise et elle inspira plusieurs études éclairant l'activité économique féminine en Grande-Bretagne. Ces nouvelles recherches ont souvent complexifié notre compréhension de la vie commerciale des femmes, et par certain côtés, remis en cause certaines des conclusions de Leonore Davidoff et Catherine Hall. Plusieurs chercheurs découvrent qu'en pratique, les femmes s'engageaient dans la vie commerciale en dépit des limites juridiques théoriques à leurs activités économiques, et en dépit du code culturel des sphères séparées ${ }^{14}$. Les travaux de Nicola Philipps et de Hannah Barker ont révélé tout un monde d'entreprises féminines à la fin du XVIII siècle ${ }^{15}$. Hannah Barker a analysé le travail de 4637 femmes citées dans les annuaires de commerce de Manchester, Leeds et Sheffield entre 1710 et 1830 . Ses recherches attestent l'importance de l'entreprise féminine et le caractère ordinaire de ces activités au XIX siècle, malgré l'influence d'une idéologie de la domesticité s'opposant au travail des femmes. Parmi les 31 entreprises de Boar Lane à Leeds en 1826, par exemple, on trouve une aubergiste, une femme tenant une manufacture d'instruments de musique, une pâtissière, une maîtresse de pension, et une vendeuse de bouchons.

L'intérêt porté, dans Family fortunes, à l'importance de la vie matérielle dans la construction de l'identité bourgeoise a aussi eu un écho dans le domaine en développement de l'histoire de la consommation et de la vie matérielle. Dans "Women and the world of goods : a Lancashire consumer and her possessions, 1751-81", Amanda Vickery étudiait la façon dont Elizabeth Shackleton, une dame de la gentry de Lancaster, faisait usage d'objets

14. Margot Finn, "Working-class women and the contest for consumer control in Victorian County Courts", Past and present, tome 161, n 1, 1998, p. 116-572; Christina De Bellaigue, Educating Women: Schooling and Identity in England and France, 1800-1867, Oxford, Oxford University Press, 2007, p. 76-84.

15. Nicola Philipps, Women in Business, 1700-1850, Londres, Boydell Press, 2006. Hannah Barker, The Business of Women: Female Enterprise and Urban Development in Northern England, 1760-1830, Oxford, Oxford University Press, 2006. 
personnels et domestiques pour créer tout un monde de signification. Par ses achats, par la façon dont elle gérait ses finances et la manière dont elle suivait les modes de la ville - toujours avec modération -, Elizabeth Shackleton s'engageait dans un processus d'autocréation qui illustrait l'influence des conceptions de la féminité à cette époque sur la vie quotidienne des femmes de la gentry, - mais aussi leurs limites ${ }^{16}$. Portant son regard sur les femmes de la petite bourgeoisie à la fin du XIX ${ }^{e}$ siècle, Chris Hosgood souligna également l'importance de la consommation. Il soutint que la façon dont ces femmes participaient aux soldes des grands magasins, leurs stratégies d'achat, contribuait à la construction d'une nouvelle identité féminine qui remettait en cause les codes de conduite que tentaient d'imposer ces magasins ${ }^{17}$. Pour Amanda Vickery et Chris Hosgood, comme pour bien d'autres, l'histoire de la consommation et de la vie matérielle permettait d'analyser l'évolution des structures sociales tout en soulignant le caractère instable et changeant des identités bourgeoises et le rôle constitutif du genre dans la création de ces identités ${ }^{18}$.

C'est notamment pour sa contribution à l'histoire de la masculinité que Family Fortunes semble encore novateur aujourd'hui. Cet ouvrage fut l'une des premières monographies de l'historiographie britannique à prêter autant d'attention à ce thème. Leonore Davidoff et Catherine Hall soulignaient le contraste entre une idée aristocratique de la masculinité, «fondée sur le sport, une conception militaire de l'honneur et trouvant son expression dans la chasse, le cheval, le cabaret et la cour faite aux femmes" et un nouvel idéal qui insistait sur le travail, la sobriété et le contrôle de soi du père de famille bourgeois ${ }^{19}$. S'inspirant en partie du travail de Leonore Davidoff et Catherine Hall, John Tosh a cherché à élargir le champ de cette branche de l'histoire sociale et culturelle du XIX ${ }^{\mathrm{e}}$ siècle qui, jusque-là, était souvent rattachée à une idée assez simpliste de la virilité victorienne (manliness) issue des grandes écoles privées (les public schools) et de l'empire. Son ouvrage, paru en 1999, A Man's Place: Masculinity and the Middle-Class Home in Victorian England ${ }^{20}$, s'intéressait surtout à la dimension construite des idéaux de la masculinité dans les rapports sociaux de sexe; il s'interrogeait sur la situation paradoxale des hommes de la bourgeoisie au XIX ${ }^{\mathrm{e}}$ siècle. Comment négocier entre une situation où, sous l'influence du renouveau évangélique,

16. Amanda Vickery, "Women and the world of goods: a Lancashire consumer and her possessions, 1751-81", dans Roy Porter et John Brewer (eds.), Consumption and the World of Goods, Londres, Routledge, 1993, p. 274-301.

17. Christopher Hosgood, "Mrs Pooter's purchase: lower middle class consumerism and the sales", dans D. Nicholls et A. Kidd (eds.), Gender, Civic Culture and Consumerism: Middle Class Identity in Britain, 18001940, Manchester, Manchester University Press, 1999, p. 146-63.

18. Pour un aperçu des publications récentes sur l'histoire de la consommation, voir Matthew Hilton, "Review Article: Class, Consumption and the Public Sphere", Journal of Contemporary History, vol. 35, n 4 , 2000, p. 655-666.

19. Leonore Davidoff et Catherine Hall, ouv. cité, p. 110.

20. John Tosh, A Man's Place: Masculinity and the Middle-Class Home in Victorian England, Londres, Yale University Press, 1999. 
la vie domestique était tenue comme une dimension constitutive de la masculinité, et où, en même temps, le foyer et l'enfance étaient conçus de plus en plus comme étant le domaine privilégié des femmes? Selon John Tosh, ces tensions suscitèrent une remise en cause de l'autorité masculine et paternelle, renforcée par une critique sans précédent de la sexualité masculine. C’est une étude nuancée et subtile, reposant sur l'analyse des archives privées de personnalités typiques de l'époque tels que Edward White Benson, proviseur de Wellington College, une des nouvelles public schools, et futur archevêque de Canterbury.

Cet intérêt nouveau pour l'histoire de la masculinité inquiétait certains chercheurs, qui y voyaient une nouvelle marginalisation de l'histoire des femmes. Mais $A$ man's place illustrait très précisément le caractère subversif de cette histoire. Comme le soulignait John Tosh en 1994, rendre visible l'homme en tant qu'individu sexué a des conséquences importantes pour tous les domaines de l'histoire, pour l'histoire de la famille, du travail, de l'entreprise, des classes sociales, des identités nationales, et aussi pour l'histoire des institutions politiques. "Pour le dire autrement, les historiens de la masculinité sont bien placés pour démontrer concrètement comment le genre est inhérent à tous les aspect de la vie sociale, même quand les femmes ne sont pas présentes ${ }^{21}$. C'est une intuition dont on mesure la justesse en découvrant le travail d'historiens de la masculinité tels que Martin Francis. Ainsi, dans un article publié en 2002, Francis proposait qu'on prenne en compte la façon dont la promotion d'un idéal masculin de self-control par trois premiers ministres conservateurs entre 1951 et 1963 avait contribué à la facilité avec laquelle la Grande-Bretagne vécut la transition de la guerre à la prospérité civile ${ }^{22}$.

\section{REPRÉSENTATIONS ET DisCOURS}

L'ouvrage de Leonore Davidoff et Catherine Hall influença donc plusieurs champs de l'histoire du genre et de l'histoire sociale pendant les années 1990. Mais l'année de sa publication correspondit aussi au moment où, sous l'influence du tournant linguistique, l'histoire sociale et culturelle prit une nouvelle orientation, accordant de l'importance à la représentation et à la signification, plutôt qu'aux catégories d'expérience et aux structures sociales qui font surtout l'objet de Family Fortunes et de ses héritiers. Pour les historiens du genre en Grande-Bretagne, comme pour ceux qui travaillent

21. John Tosh, "What should historians do with masculinity? Reflections on nineteenth-century Britain", History Workshop Journal, tome 38, n 1, 1994, p. 179-180.

22. Martin Francis, "Tears, tantrums and bared teeth: the emotional economy of three Conservative Prime Ministers, 1951-1963", Journal of British Studies, tome 41, n 3, 2002, p. 354-387. 
aux États-Unis, l'article de Joan Wallach Scott qui parut dans la American Historical Review en 1986, eut une importance décisive ${ }^{23}$.

Dans "Gender as a category of historical analysis", Scott prenait ses distances avec le concept du genre tel qu'il était compris par Leonore Davidoff et Catherine Hall, c'est-à-dire comme touchant surtout aux rapports sociaux des sexes et à la division sexuelle du travail. Plus important pour Scott était la construction discursive du genre comme catégorie sociale. De plus, le genre, selon elle, était surtout important comme "un des moyens principaux de signifier les rapports du pouvoir» ${ }^{24}$. Pour Joan Scott, au lieu de reconstruire des expériences d'hommes et de femmes du passé, les historiens du genre devaient retracer la construction des discours du genre et la diffusion de ces idées. Elle allait même plus loin : en reconnaissant le caractère discursif du genre, écrivait-elle, le chercheur est confronté à l'impossibilité de découvrir l'expérience réelle, car toute expérience sera sujette à ces constructions discursives. Cette position extrême, qui remet en cause la catégorie de l'expérience comme objet de recherche historique, séduit moins en Grande-Bretagne qu'aux États-Unis. Outre-Manche, l'influence du féminisme socialiste se fait plus sentir, et rattache l'histoire des femmes et l'histoire du genre à une tradition qui souligne l'importance de la vie matérielle. D'autre part, la distance entre l'approche de Joan Scott et celle de Leonore Davidoff et Catherine Hall était peut-être moins grand qu'il ne semblait à l'époque; Family Fortunes s'intéressait après tout à la construction discursive du genre pour son influence sur la hiérarchie sociale. Néanmoins, au début des années 1990, la démarche de Joan Scott semblait signaler un nouveau départ dans l'histoire du genre, et plusieurs publications s'intéressèrent surtout à l'histoire politique du genre, à la façon dont la représentation des différences sexuelles construisent et expriment les rapports de pouvoir.

Ainsi, Britons de Linda Colley, un ouvrage qui eut à la fois un grand succès public et une influence importante dans le monde universitaire, soulignait comment le genre était au centre de la nouvelle identité britannique qui se construisit dans le siècle qui suivit l'acte d'union avec l'Ecosse ${ }^{25}$. Pour Linda Colley, cette nouvelle identité était fondée sur un idéal de la masculinité britannique et protestant, défini contre «l'autre», celui d'une France catholique et efféminée. Une notion de féminité britannique, centrée sur le "home», creuset du patriotisme, renforçait cet idéal masculin du caractère national. En même temps, en reconnaissant cette identité patriotique féminine, Linda Colley était amenée à discerner les activités politiques de femmes théoriquement exclues de la vie publique. Elle reprenait ainsi l'accent mis par

23. Joan Wallach Scott, "Gender, a useful category of historical analysis", American Historical Review, tome $91, \mathrm{n}^{\circ}$ 5, 1986, p. 1052-1075, traduction française "Genre : une catégorie utile d'analyse historique», Le genre de l'histoire, Les cahiers du GRIF, 37/38, 1988, p. 125-153.

24. Idem, p. 1067

25. Linda Colley, Britons: Forging the Nation, 1707-1837, New Haven, Yale University Press, 1992. 
Leonore Davidoff et Catherine Hall sur l'importance d'une idéologie de la domesticité; mais en soulignant la construction discursive de cette idéologie, elle en soulignait les limites pratiques et attirait l'attention sur la dimension politique du genre.

Cette façon de souligner l'influence et le rôle politique des représentations sexuées fut prolongée dans le travail d'Anna Clark, une historienne américaine dont le travail a eu un écho parmi les historiens britanniques du genre. Dans The Struggle for the Breeches, Anna Clark revisitait le travail d'E.P. Thompson pour démontrer comment les représentations sexuées étaient au cœur de la nouvelle identité plébéienne au début du XIX ${ }^{\mathrm{e}}$ siècle ${ }^{26}$. Elle prétendait que c'est par l'adoption d'un idéal de la domesticité que les chartistes parvinrent à lancer le dialogue avec le libéralisme victorien qui assura la réalisation de certains de leurs objectifs au cours du siècle. En effet, c'est en se rattachant à un idéal masculin du père de famille sobre et chef de foyer respectable, que les hommes des classes populaires ont pu prendre place dans le système politique britannique. De plus, Anna Clark avançait que l'extension progressive du droit de vote au cours du XIX ${ }^{\mathrm{e}}$ siècle avait été rendu possible par l'exclusion des femmes du suffrage et par une définition masculine de la citoyennetée ${ }^{27}$.

Cet intérêt pour la manière dont les représentations sexuées influencent l'histoire politique a aussi inspiré les travaux de Jon Lawrence et de David Jarvis sur le parti conservateur. Lawrence montre comment, à la fin du XIX ${ }^{\mathrm{e}}$ siècle, c'est en partie en exploitant une vision particulière de la masculinité populaire, que les Tories parvinrent à gagner le soutien des nouveaux électeurs de la classe ouvrière. Ceux-ci, auparavant plus réceptifs aux Libéraux (qui, comme le montre Clark, avaient des liens avec les partis plébéiens du début du siècle), furent séduits par un discours défendant "les plaisirs traditionnels de l'homme", distinguant les Tories d'un parti Libéral souvent hostile à la culture du public house et des sports plébéiens ${ }^{28}$. S'intéressant à la période de l'entre-deux-guerres, David Jarvis montra comment les conservateurs, se sentant en position affaiblie après l'introduction du suffrage universel en 1918 et 1928, et avec la montée du parti travailliste, changèrent de stratégie. Cessant d'insister sur cet idéal de la masculinité populaire, ils tentèrent d'attirer le vote féminin en se présentant comme le parti de l'économie

26. Anna Clark, The Struggle for the Breeches: Gender and the Making of the British Working Class, Berkeley, University of California Press, 1995.

27. Anna Clark, "Gender, class and the constitution: franchise reform in England, 1832-1928", dans James Vernon (ed.), Re-Reading the Constitution: New Narratives in the Political History of England's Long Nineteenth Century, Cambridge, Cambridge University Press, 1996, p. 230-53. Rappelons qu'en Grande-Bretagne, les réformes successives de 1832, 1867 et 1884 donnèrent le droit de vote à de nouvelles couches d'hommes (successivement ceux de la middle class, les ouvriers qualifiés et les travailleurs agricoles). En revanche, les femmes, explicitement écartées du suffrage pour la première fois en 1832, n'obtinrent le droit de vote qu'en 1918, et encore si elles avaient plus de 30 ans (21 ans en 1928).

28. Jon Lawrence, "Class and gender in the making of urban Toryism, 1880-1914", English Historical Review, tome $108, \mathrm{n}^{\circ} 428,1993$, p. 629-653. 
domestique, responsable et patriotique ${ }^{29}$. Cette image du bon sens féminin est opposée à l'idée d'une masculinité ouvrière irresponsable et sensible aux séductions du socialisme. Ce sont des images qui exploitent les distinctions traditionnelles des rôles masculins et féminins dans la famille ouvrière. Ces références s'appuient sur un idéal puissant de la domesticité, et soulignent habilement les tensions entre le socialisme et le conservatisme fondamental de la culture populaire. Pour Jon Lawrence et pour David Jarvis donc, comme pour Anna Clark, il était essentiel de reconnaître la façon dont le discours du genre influence et colore le discours politique.

La politique du genre est également au centre d'un nouvel intérêt pour l'histoire impériale et postcoloniale. S'inspirant des idées de Edward Said et Homi Bhabha, une nouvelle génération d'historiens travaillant sur l'empire britannique tente de mieux comprendre les politiques d'exclusion et d'inclusion du monde impérial. C'est une approche qui attire particulièrement les chercheurs travaillant aux États-Unis, mais qui influence aussi l'historiographie britannique ${ }^{30}$. Nous retrouvons à nouveau Catherine Hall. Dans Civilizing Subjects : Metropole and Colony in the English Imagination, 1830-1867 (2002), elle propose une étude approfondie des relations entre la Jamaïque et la métropole qui souligne comment la notion de citoyenneté dans la Grande-Bretagne victorienne dépendait d'un idéal de la masculinité soulignant l'interaction entre genre et race ${ }^{31}$. Catherine Hall soutient que les années 1850 virent la disparition d'une certaine conception de la différence des races provenant des milieux missionnaires - conception selon laquelle les races africaines étaient sensibles à un processus de civilisation. Face à l'intransigeance de la population jamaïcaine envers les efforts faits par les missionnaires pour lui inculquer leurs mœurs européennes, ceux-ci eurent recours à un discours qui soulignait plutôt le contraste entre les vertus mâles de l'homme britannique et la paresse efféminée des Jamaïcains. Au cours des années 1860, ces différences furent de plus en plus souvent conçues comme des catégories biologiques. Pour Catherine Hall, cette conception sexuée de la différence biologique soulignait et renforçait la vision exclusivement masculine de la citoyenneté identifiée par Anna Clark et d'autres.

Comme le montrent les travaux de Linda Colley, de Jon Lawrence, de David Jarvis et de Catherine Hall, l'insistance de Joan Scott sur l'importance du genre comme moyen de signifier les rapports de pouvoir eut une influence profonde sur l'analyse de la participation et de la communication

29. David Jarvis, "Mrs Maggs and Betty: the Conservative appeal to women voters in the 1920s", Twentieth Century British History, tome 5, n 2, 1994, p. 129-152.

30. Voir par exemple, Antoinette Burton, Burdens of History: British Feminists, Indian Women, and Imperial Culture, 1865-1915, Chapel Hill, University of North Carolina Press, 1994; Ann Laura Stoler, Carnal Knowledge and Imperial Power: Race and the Intimate in Colonial Rule, Berkeley, University of California Press, 2002.

31. Catherine Hall, Civilizing Subjects: Metropole and Colony in the English Imagination, 1830-1867, Oxford, Polity Press, 2002. 
politique, elle provoqua une réévaluation profonde de l'histoire de l'identité nationale et de la citoyenneté.

\section{IDENTITÉ, DISCOURS ET EXPÉRIENCE}

Au cours des années 1990, on a donc vu les historiens du genre en Grande-Bretagne suivre deux tendances principales, l'une soulignant l'importance du genre dans l'analyse de la vie sociale, l'autre s'intéressant surtout aux discours sexués et à leur influence. Pendant cette même période, le genre a été de plus en plus accepté comme catégorie d'analyse par ceux qui ne se considèrent pas d'abord comme historiens du genre, et les travaux des spécialistes ont commencé à avoir une importance significative en-dehors des études spécialisées. Par exemple, une des conséquences de l'attention portée par Linda Colley au rôle public des femmes patriotes a été la prolifération d'ouvrages sur les activités politiques de femmes, qui, en principe, étaient exclues du monde publique. Les travaux de chercheuses comme Kathryn Gleadle, Sarah Richardson et Kim Reynolds, qui soulignaient l'importance du rôle des femmes sur les marges de la vie publique, firent à leur tour découvrir combien la vie politique, conçue plus largement, se conduit de façon informelle et en dehors des canaux officiels ${ }^{32}$.

Cependant, parmi la nouvelle génération de spécialistes de l'histoire du genre, une attitude critique s'est manifestée envers ces deux tendances dominantes. Pour certains, c'est l'antihumanisme inhérent à l'analyse des discours qui pose problème. Pour d'autres, une histoire qui s'attache à l'expérience sans élucider l'action du discours sur l'individu est insuffisante.

Parmi les critiques de l'histoire des représentations, et de l'histoire de l'expérience féminine telle qu'elle était conçue dans les années 1980, figure Amanda Vickery, qui publia The Gentleman's Daughter: Women's Lives in Georgian England en $1998{ }^{33}$. Développant son étude de la vie d'Elizabeth Shackleton, Amanda Vickery élargissait son analyse à la vie des femmes de la gentry au XVIII e siècle. Elle s'appuyait sur la lecture des lettres, des journaux intimes, des livres de comptes de ces dames pour contester toute vision trop misérabiliste de l'idée des sphères séparées : selon Amanda Vickery, ces dames avaient une conception du mariage alliant affection et prudence pragmatique. Elles prennent plaisir et sont fières de leur habileté en tant que maîtresses de maison, et loin d'être enfermées à l'intérieur, ces femmes habitent un monde social hétérogène, bien moins clairement divisé entre public et privé

32. Kathryn Gleadle et Sarah Richardson (eds.), Women in British Politics, 1760-1860: the Power of the Petticoat, Basingstoke, Macmillan, 2000; Kim Reynolds, Aristocratic Women and Political Society in Early-and Mid-Victorian Britain, Oxford, Clarendon Press, 1998.

33. Amanda Vickery, The Gentleman's Daughter: Women's Lives in Georgian England, Londres, Yale University Press, 1998. 
qu'on ne le penserait en s'appuyant sur la littérature prescriptive. Convaincue de l'importance de l'expérience individuelle, Amanda Vickery tentait à la fois de remettre en cause l'idée que l'idéologie domestique représente toujours une contrainte sur la vie des femmes, et de mieux comprendre la contribution de ce discours à la formation de l'identité sociale et de la subjectivité de ces femmes. Pour elle, prendre conscience de l'interaction entre discours culturel et processus matériels permettait de reconnaître que l'expérience des femmes peut mener à la transformation des mentalités et des discours. Avec The Gentleman's Daughter, elle tentait de résoudre le problème du rapport entre genre, identité, individu et subjectivité.

Pour Michael Roper, l'histoire des émotions devait résoudre ce problème. Tout en reconnaissant l'influence et la force des idées de Joan Scott sur les relations de pouvoir et les représentations, il était déçu par cette tendance de l'histoire culturelle qui, en privilégiant l'analyse des discours, réduisait les émotions et l'expérience de l'individu à une sorte de code linguistique. "Trop souvent, ce qui fait défaut dans ces analyses linguistiques c'est une reconnaissance suffisante de la dimension concrète, des pratiques de la vie quotidienne, de l'expérience humaine formée par la relation affective avec d'autres, et de l'expérience comme processus continu - conscient et inconscient - de gestion des émotions à l'égard des autres " ${ }^{34}$. Pour Michael Roper, la voie à adopter devrait être celle d'une histoire influencée par la psychanalyse afin de comprendre les mobiles des acteurs de l'histoire. Une approche biographique, informée par la psychanalyse, nous amènerait ainsi à comprendre les codes culturels du genre comme des constructions, ainsi que la façon dont ces codes se rattachent aux expériences vécues et aux réponses émotionnelles à ces expériences.

L'objectif de ces historiens du genre est donc d'essayer de mieux comprendre l'interaction entre les codes culturels du genre et l'expérience vécue de l'individu. L'historiographie tentant surtout de capturer les voix de celles qui étaient traditionnellement ignorées par l'histoire, et travaillant souvent avec une vision quelque peu simpliste de "la femme» comme sujet historique, a laissé la place à des recherches qui font appel à une conception bien plus complexe et multiple de l'identité sexuée, et qui insistent sur le genre comme catégorie essentielle de l'analyse historique. Ceci est aujourd'hui largement accepté même en dehors des études spécialisées sur le genre. Les publications récentes de Oxford University Press témoignent de l'emprise du genre dans l'histoire du commerce, de l'économie, de la politique. Dans l'analyse des divers phénomènes et changements qui font l'objet de leur étude, Frank Trentmann, Martin Daunton, Norman McCord et Bill Purdue prennent

34. Michael Roper, "Slipping out of view: subjectivity and emotion in gender history", History Workshop Journal, tome 59, n 1,2005 , p. 62. 
tous en compte les différences entre les expériences féminine et masculine, et la façon dont le genre structure les relations de pouvoir ${ }^{35}$.

Cependant, malgré les efforts d'historiens comme Amanda Vickery et Michael Roper, il me semble que la question des rapports entre l'individu, l'expérience et les discours reste ouverte. Sans une explication des liens entre l'individu et le social, il est difficile de comprendre le changement dans le temps des constructions discursives et de l'expérience des hommes et des femmes. Je travaille sur l'histoire de l'éducation et de l'adolescence au XIX ${ }^{e}$ siècle, et la question du rapport entre la vie des jeunes gens et leur apprentissage des normes de la féminité et la masculinité, la question des rapports entre discours, expérience et conscience de soi me paraît essentielle. Mais le travail d'Amanda Vickery, malgré toute sa subtilité et son évocation habile de la façon dont les femmes de la gentry anglaise négociaient leur rôle en répondant aux normes courantes de la féminité, n'a pas pu répondre d'une façon satisfaisante à la question de savoir comment ces normes ont pu évoluer au courant du XVIII siècle. En soulignant la façon dont ces femmes de la gentry répondaient individuellement aux normes sociales, et en minimisant l'importance des discours normatifs, Amanda Vickery nous présente un portrait curieusement statique et immobile. D'un autre côté, il me semble que l'approche de Michael Roper, reposant sur la psychanalyse, risque toujours l'anachronisme en imposant au passé des catégories contemporaines, ou en supposant une continuité anhistorique des émotions. Il me semble que l'histoire de l'enfance - qui s'intéresse non seulement à l'évolution des rapports familiaux, mais aussi à la façon dont le langage et l'interprétation des sentiments ont pu changer - souligne tout particulièrement ces risques.

Revenons-nous à un débat analogue à celui de la fin des années 1970, entre les historiennes convaincues de la continuité du régime patriarcal et celles qui voyaient dans le concept de patriarcat un obstacle à l'analyse du changement et de la diversité des rôles sexuels? Ne pourrait-on voir dans les débats des 1970, comme dans les ouvrages de Michael Roper et Amanda Vickery, les mêmes tensions autour des questions de continuité et de changement, ou de relations entre l'individu et la société? Ce qui a surtout changé, entre les années 1970 et le début du XXIe siècle, c'est que l'on reconnaît maintenant que les questions posées par les historiens du genre se situent au cœur même de l'entreprise historique. Grâce en partie aux découvertes de Leonore Davidoff et Catherine Hall, de Linda Colley, de John Tosh, on reconnaît désormais que le genre, comme la race ou la classe sociale, est le fruit de processus qui sont à la fois sociaux et discursifs. Il me semble qu'aujourd'hui

35. Frank Trentmann, Free Trade Nation. Commerce, Consumption and Civil Society in Modern Britain, Oxford, Oxford University Press, 2008; Martin Daunton, Wealth and Welfare: an Economic and Social History of Britain 1851-1951, Oxford, Oxford University Press, 2007; Norman McCord et Bill Purdue, British History 1815-1914, Oxford, Oxford University Press, 2007. 
l'histoire du genre en Grande-Bretagne se trouve à nouveau à un moment de transition. Pour progresser, et pour mieux comprendre les rapports entre le discours et le social, il faudra s'assurer que nos études s'appuient sur une analyse plus sensible aux multiples interdépendances du genre avec la classe, la religion, l'économie, la politique, l'ethnicité et la subjectivité.

Christina de Bellaigue est lecturer en histoire contemporaine à l'université d'Oxford 